\title{
Computerized Performance Monitoring Systems: Learning and Living with its Limitations
}

\author{
Daniel J. Luchins
}

Published online: 30 March 2007

(C) Springer Science+Business Media, LLC 2007

\section{Erratum to: Adm Policy Ment Health and Ment Health Serv Res DOI 10.1007/s10488-006-0092-9}

The following errors were inadvertently made:

Page 3 line 11 the sentence beginning "An initiating" is completely garbled. It should read: "An example of such an indicator would be a thyroid screen within 6 months prior to starting lithium, or a serum lithium level 5 to 10 days after initiating or increasing lithium dosage. The reports gave the statewide average for each indicator, the facility average, each physician's average, and listed each instance when a particular physician failed to order a particular laboratory test on a specific patient."

Page 4 line 7 from the bottom The sentence "Since no physician remained,' is out of place. It belongs at the end of the first paragraph on page 5 line 3 before adding the sentence from page 3 "Since no physician remained" the following words need to be added to the "these same physicians" "had raised their scores, often into the 90$100 \%$ range."
The whole section would read like this:

Fourth, the valence of an indicator can be changed by the very fact that it is monitored and this information feedback to the clinicians. Let me make reference to our earlier work on whether physicians were correctly ordering laboratory tests. Initially, many of our system's least able physicians scored in the $40-60 \%$ ranges, so there was a positive relationship between scores on this index and my estimation of clinical skills. But after 6 months of feedback from central office to their medical directors, these same physicians had raised their scores, often into the $90-100 \%$ range. Since no physicians remained in the $40-60 \%$ range, and since (as I previously noted) scores in the $90-100 \%$ range reflected routinized and not clinically informed decision making, there was now a reversal of the previously noted positive relationship between performance on this indicator and my own estimation of physician skill.

The online version of the original article can be found at http://dx.doi.org/10.1007/s10488-006-0092-9

D. J. Luchins $(\bowtie)$

Department of Psychiatry, University of Chicago, 5841, S.

Maryland Ave. MC 3077, Chicago, IL 60637, USA

e-mail:danl@yoda.bsd.uchicago.edu 